\title{
Fatores de Risco para Síndrome Metabólica em Cadeirantes - Jogadores de Basquetebol e Não Praticantes
}

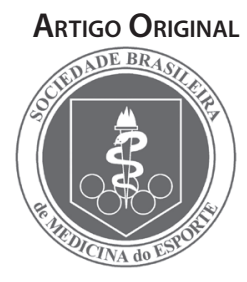

\author{
Risk Factors for the Metabolic Syndrome in Wheelchair Users - \\ Basketball Players and Non-Practitioners.
}

\author{
Rafael Quintana' \\ Cassiano Merussi Neiva² \\ 1. Faculdade de Ciências da Saúde \\ Centro Universitário de Patos de \\ Minas - UNIPAM Patos de Minas \\ / MG \\ 2. Faculdade de Ciências \\ Universidade Estadual Paulista Júlio \\ de Mesquita Filho - UNESP Bauru \\ Bauru / SP
}

Endereço para correspondência: Rua João XXIII, 20/203 - Sobradinho - CEP 38701-126 - Patos de Minas/ MG - Brasil

Email: rafaelquint@terra.com.br

Submetido em 08/06/2006 Versão final recebida em 21/08/2007 Aceito em 03/01/2008

\begin{abstract}
RESUMO
A atividade física tem sido sistematicamente estudada como fator preventivo no acometimento de enfermidades crônico-degenerativas, especialmente a síndrome metabólica e doenças cardiovasculares. Sedentarismo está relacionado à diminuição ou ausência de parâmetros mínimos de exigência física diária além do estado de repouso, sendo este fortemente associado à redução na condição de saúde dos indivíduos portadores de deficiência física. As respostas metabólicas do organismo mediante a ausência de determinada musculatura, ou sua inatividade pela falta de estímulos, conduzem a diferenças significativas na estruturação da composição corporal. Esta pesquisa teve como objeto o exercício físico regular e a condição de saúde de indivíduos portadores de deficiência física, através da determinação do perfil antropométrico e bioquímico sangüíneo, e ainda pela determinação da prevalência de fatores de risco para síndrome metabólica. Foram estudados 27 homens com paraplegia (T2-L1), portadores de seqüelas de poliomielite ou amputados, divididos em jogadores de basquetebol cadeirantes (JBC) e não jogadores de basquetebol cadeirantes (NJBC). Os JBC apresentaram circunferência de cintura menor comparada aos $\mathrm{NJBC}, 76,40 \pm 8,44$ e $89,25 \pm 9,73 \mathrm{~cm}$ respectivamente $(p<0,05)$. A pressão arterial sistólica foi significativamen-

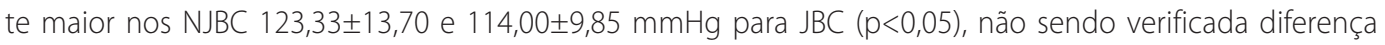
para PAD. Os NJBC apresentaram valores superiores aos JBC para a bioquímica sangüínea de glicemia, TG, $C T$ e frações, exceto para HDL-C $(p<0,05)$. Os indivíduos NJBC apresentaram alta prevalência para fatores de risco da SM, sendo hipertensão arterial prevalente em 58,33\% dos indivíduos, dislipidemia de HDL-C presente em $50 \%$ e circunferência de cintura acima da normalidade em $41,66 \%$. Os achados do presente estudo sugerem um papel importante do exercício físico na prevenção da síndrome metabólica, embora estudos adicionais devam ser realizados para a melhor compreensão dos mecanismos que promovem a saúde dos deficientes físicos ativos.
\end{abstract}

Palavras-chave: atividade física, promoção da saúde, deficiência física, síndrome metabólica.

\begin{abstract}
Physical activity has been systematically studied as a prevention element of chronic degenerative diseases, especially the metabolic syndrome and cardiovascular disease. Sedentary habits are related to the decrease or absence of minimal parameters of daily physical exercises beyond the resting condition, and they have been strongly associated with the decrease of health in disabled individuals. The metabolic responses of the body in the absence of certain muscles, or its inactivity due to the lack of stimuli, lead to significant differences in the structure of the body composition. This research had as aim regular physical exercise and health condition of disabled individuals, through the determination of the anthropometric and blood biochemical profile. Moreover, the prevalence of risk factors for the metabolic syndrome has been determined. 27 men with paraplegia (T2-L1), presenting polio sequelas or amputation, were divided in two groups: wheelchair basketball players and (WBP) and wheelchair non-basketball players (WNBP). The WBP presented waist circumference smaller than the WNBP, $76.40+8.44$ and $89.25+9.73 \mathrm{~cm}$ respectively $(p<0.05)$. The systolic arterial pressure was significant bigger in WNBP $123.33+13.70$ and $144.00+9.85 \mathrm{mmHg}$ for WBP $(p<0.05)$, and no difference for PAD was verified. WNBP presented higher numbers than WBP for blood glycemic biochemistry, TG, CT and fractions, except for HDL-C ( $p<0.05)$. WNBP individuals presented high prevalence for risk factors of metabolic syndrome, being arterial hypertension prevalent in $58.33 \%$ of the individuals; HDL-C dyslipidemia present in 50\%, and waist circumference above normality in $41.66 \%$. The findings of the present study corroborate others described in the literature on high prevalence of risk factors for metabolic syndrome among individuals with physical disabilities with low levels of regular physical activity. The evidence raised suggests an important role of regular physical exercise as suitable prevention device for metabolic syndrome.
\end{abstract}

Keywords: physical activity, health promotion, physical disability, metabolic syndrome. 


\section{INTRODUÇÃO}

Entre os indivíduos portadores de deficiência física, alterações bioquímicas e metabólicas indesejáveis se estabelecem com a inatividade física, resultando em redução da massa muscular e acúmulo excessivo de tecido adiposo corporal, situação verificada através de parâmetros antropométricos ${ }^{(1-4)}$.

Tais alterações, especialmente no nível de deposição de gordura nos adipócitos, estão descritas na literatura como fator de risco para obesidade, dislipidemia, diabetes, síndrome metabólica e doenças cardiovasculares, desfavorecendo a saúde dos indivíduos portadores de deficiência física sedentários ${ }^{(5-9)}$.

Os benefícios gerados através da prática regular de atividade física sob a redução dos riscos de mortes prematuras por acometimento de doenças cardiovasculares, câncer, diabetes e síndrome metabólica, estão satisfatoriamente descritos na literatura científica ${ }^{(10-16)}$.

Os estudos na população brasileira de deficientes físicos sobre síndrome metabólica e exercício físico ainda são escassos, considerando a dificuldade de uma amostra significativa e a diversidade dos quadros de incapacidades.

Portanto, o objeto de estudo deste trabalho é o exercício físico regular e sua influência na promoção da saúde de indivíduos com deficiência física, tendo como objetivo a descrição antropométrica e bioquímica sangüínea de indivíduos portadores de lesão física, comparando grupos de jogadores de basquetebol em cadeira de rodas e de não jogadores. E ainda, analisar estes dados identificando a prevalência de fatores de risco para síndrome metabólica.

\section{MATERIAIS E MÉTODOS}

\section{Amostra}

Para o estudo descritivo de coorte transversal da população de indivíduos portadores de deficiência física, adotou-se como critério de inclusão, portadores de paraplegia, amputações de membros inferiores e seqüelas de poliomielite. A amostra foi composta de 27 homens, sendo 14 lesados medulares (T2-L1), 5 amputados e 8 com seqüelas de poliomielite, obtida por demanda espontânea entre duas equipes de jogadores de basquetebol em cadeira de rodas e por contato telefônico na base de cadastro da Associação dos Deficientes do município de Patos de Minas. Foram estabelecidos dois grupos, sendo 15 jogadores de basquetebol cadeirante (JBC), com pratica superior a 12 meses, freqüência semanal de duas a três vezes e tempo de exercício por sessão de duas horas, e o outro grupo de não jogadores de basquetebol cadeirante (NJBC), composto por 12 indivíduos.

Todos os participantes do estudo concordaram de forma voluntária com sua participação no mesmo e a divulgação dos resultados após leitura e assinatura do Termo de Consentimento Livre Esclarecido, tendo sido mantido o anonimato de todos. Foram seguidas rigorosamente as normas da Declaração de Helsinki e as precípuas da Lei CNS 196/96, sendo este aprovado pelo Comitê de Ética e Pesquisa da Universidade de Franca sob o protocolo no 194/05.

\section{Antropometria}

A estatura foi mensurada por estadiômetro marca Sanny ${ }^{\circledR}$ com acurácia de 0,1 mm, fixado horizontalmente a $40 \mathrm{~cm}$ de uma base rígida. $A$ massa corporal foi obtida em uma balança digital Filizola ${ }^{\circledR}$, com acurácia de 0,100 g, utilizando-se uma base rígida de madeira e cadeira de rodas quando necessário, determinando-se a massa corpórea por diferença. As próteses utilizadas pelos amputados foram pesadas separadamente, determinando também por diferença a massa corporal.

Para a medida de circunferência da cintura foi utilizada uma fita métrica flexível e não elástica marca Mabis ${ }^{\circledR}$, com mecanismo de re- tração e graduação de 0,1 mm, registrando-se a medida para o meio centímetro mais próximo. $\mathrm{O}$ avaliado estando com o tronco desnudo e posicionado sentado e/ou deitado, determinou-se à circunferência da cintura em triplicata, considerando a média entre as medidas. Como referencial para tomada da medida foi adotado o ponto coincidente com a distância média entre a última costela e a crista-ilíaca após uma expiração normal, sem a compressão da pele ${ }^{(17)}$.

\section{Variável hemodinâmica}

A pressão arterial foi obtida por método de ausculta com a utilização de estetoscópio, modelo Tycos ${ }^{\circledR}$ e esfigmomanômetro coluna de mercúrio modelo Missouri ${ }^{\circledR}$, aferidos pelo INMETRO. A medida foi dada em milímetros de mercúrio ( $\mathrm{mmHg}$ ) observando-se as recomendações da Sociedade Brasileira de Cardiologia ${ }^{(18)}$. Para determinação da pressão arterial sistólica (PAS) foi considerada a fase I de Korotkoff, e a fase $\mathrm{V}$ para a pressão arterial diastólica (PAD) ${ }^{(19)}$.

\section{Bioquímica sangüínea}

Na obtenção da amostra sangüínea foram observadas 12 horas de jejum, sem consumo de bebidas alcoólicas pelo período de 72 horas e repouso de 30 minutos antes da coleta no período da manhã.

As análises bioquímicas da glicemia de jejum, triglicerídios (TG), colesterol total (CT) e frações deste, HDL-C, LDL-C e VLDL-C, foram realizadas em triplicata, considerando a média entre elas. Foram consideradas as diretrizes para preservação da amostra de glicose $^{(20)}$.

As análises foram obtidas com a utilização de kits de reagentes Liquiform enzimáticos e controle Qualitrol ${ }^{\circledR}$ da LabTest ${ }^{\circledR}$. O equipamento Express Plus modelo 550 da Bayer ${ }^{\circledR}$ foi utilizado para leitura das amostras. O método de determinação da glicose adotado foi o enzimático colorimétrico da oxidase. Para o colesterol total, triglicerídios e HDL-C foram aplicados o método colorimétrico enzimático. As frações de colesterol LDL-C e VLDL-C foram obtidas pela fórmula de Friedewald(21).

\section{Tratamento estatístico}

Para as análises estatísticas foi utilizado o programa SPSS 13.0 for Windows ${ }^{\circledR}$, verificando a distribuição normal da amostra pela aplicação do teste Shapiro-Wilk, com coeficiente de significância superior a 0,05 para distribuição normal. Foram feitas análises estatísticas descritivas e para a comparação de médias entre duas amostras independentes utilizou-se o teste $t$ de student com $p<0,05^{(22)}$.

\section{RESULTADOS}

Os vinte e sete indivíduos estudados apresentaram idade média

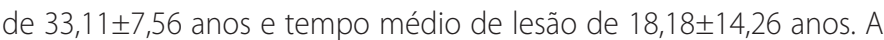
média de idade no grupo JBC foi inferior comparada ao grupo de NJBC $30,13 \pm 6,32$ e 36,83 $\pm 7,56$ anos de idade respectivamente $(p<0,05)$. Os indivíduos não jogadores apresentaram tempo de lesão médio de $22,7 \pm 17,1$ anos, superior aos jogadores $14,5 \pm 10,7$ anos $(p<0,05)$.

Significativamente inferior também foi a medida circunferencial da

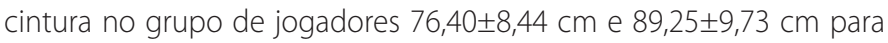
os não-jogadores $(p<0,05)$. Segundo critérios da Sociedade Brasileira de Cardiologia - SBC (23) alterações da circunferência de cintura foram observadas em 41,66\% dos NJBC, estando os demais dentro dos valores de normalidade.

Na amostra estudada foi evidenciada diferença estatisticamente significativa $(p<0,05)$ para a PAS entre os grupos $114,00 \pm 9,85 \mathrm{e}$ $123,33 \pm 13,70 \mathrm{mmHg}$ respectivamente, não sendo significativa a diferença para PAD. Sob os critérios de classificação da hipertensão arterial da SBC (18), 86,66\% dos indivíduos JBC a PA encontrava-se com valores ótimo ou normal, dentre estes, 6,66\% apresentaram valores limítrofes para normalidade. $\mathrm{O}$ quadro de hipertensão em estágio leve foi pre- 
valente em 6,66\%. No grupo de NJBC, 41,66\% apresentavam pressão arterial normal ou ótima, com prevalência de valores limítrofes de normalidade em 33,33\%. Quadros de hipertensão arterial leve e severa representaram $24,99 \%$.

Nas dosagens de glicose, CT, VLDL-C, LDL-C e triglicerídios foram observadas diferenças significativas entre os grupos, não sendo verificada diferença na fração de colesterol HDL-C (tabela 1).

Tabela 1. Característica da amostra para glicose, CT, TG, HDL-C, LDL-C e VLDL-C

\begin{tabular}{l|c|c|c|c|c}
\hline \multirow{2}{*}{ Variável } & \multicolumn{2}{|c|}{ JBC $(\boldsymbol{n}=15)$} & \multicolumn{2}{c|}{ NJBC $(\boldsymbol{n}=12)$} & \multirow{2}{*}{$\boldsymbol{p}$} \\
\cline { 2 - 5 } & Média & $\begin{array}{c}\text { Desvio } \\
\text { padrão }\end{array}$ & Média & $\begin{array}{c}\text { Desvio } \\
\text { padrão }\end{array}$ & \\
\hline Glicose & 87,48 & $\pm 6,48$ & 95,77 & $\pm 11,62$ & $0,027^{*}$ \\
\hline CT & 154,73 & $\pm 24,87$ & 199,13 & $\pm 37,87$ & $0,001^{*}$ \\
\hline TG & 96,57 & $\pm 35,20$ & 131,33 & $\pm 47,07$ & $0,038^{*}$ \\
\hline HDL-C & 45,73 & $\pm 10,35$ & 45,00 & $\pm 11,19$ & 0,862 \\
\hline LDL-C & 89,68 & $\pm 24,98$ & 127,87 & $\pm 37,60$ & $0,004^{*}$ \\
\hline VLDL-C & 19,31 & $\pm 7,04$ & 26,26 & $\pm 9,41$ & $0,038^{*}$ \\
\hline
\end{tabular}

${ }^{*} p<0,05$

Adotando como referência os critérios da American Diabetes Association ${ }^{(24)}$ para determinação do diabetes, verificou-se que $88,88 \%$ dos indivíduos de ambos os grupos apresentavam nível glicêmico dentro da normalidade, e 11,11\% sob níveis inapropriados.

Foram constatadas alterações lipidêmicas em ambos os grupos, pelos valores de referência da III Diretrizes sobre Dislipidemia da SBC (25). Entre os JBC pode ser verificada dislipidemia com redução do nível de HDL-C em 40\% dos indivíduos, não sendo verificado qualquer outro quadro dislipidêmico. A hipertrigliceridemia prevaleceu em 8,33\% dos NJBC e 16,66\% apresentaram hipercolesterolemia. Neste mesmo grupo foram observados aumento de LDL-C em 16,66\% e redução de HDL-C em $50 \%$.

Considerando os componentes adotados pela SBC(23) para a síndrome metabólica nas diretrizes para diagnóstico e tratamento, os NJBC apresentaram dados que sugerem uma alta prevalência dos fatores de risco, destacando-se a hipertensão arterial, obesidade central e dislipidemia, sendo está última pronunciada também entre os JBC (tabela 2).

Tabela 2. Prevalência de fatores de risco para síndrome metabólica

\begin{tabular}{l|c|c|c|c|c|c}
\hline \multirow{2}{*}{ Componente } & \multicolumn{2}{|c|}{$\begin{array}{c}\text { JBC } \\
\mathbf{n}=15\end{array}$} & \multicolumn{2}{c|}{$\begin{array}{c}\text { NJBC } \\
n=12\end{array}$} & \multicolumn{2}{c}{$\begin{array}{c}\text { Total } \\
\mathbf{n}=27\end{array}$} \\
\cline { 2 - 7 } & $\mathbf{n}$ & $\%$ & $\mathbf{n}$ & $\%$ & $\mathbf{n}$ & $\%$ \\
\hline $\begin{array}{l}\text { Circunferência de cintura } \\
(>102,0 \mathrm{~cm})\end{array}$ & - & - & 5 & 41,66 & 5 & 18,51 \\
\hline $\begin{array}{l}\text { HDL-C } \\
(<40 \mathrm{mg} / \mathrm{dl})\end{array}$ & 6 & 40,00 & 6 & 50,00 & 12 & 44,44 \\
\hline $\begin{array}{l}\text { TG } \\
(\geq 150 \mathrm{mg} / \mathrm{dl})\end{array}$ & 1 & 6,66 & 2 & 16,66 & 3 & 11,11 \\
\hline $\begin{array}{l}\text { PAS e PAD } \\
(\geq 130 \mathrm{e} \geq 85 \mathrm{mmHg})\end{array}$ & 1 & 6,66 & 7 & 58,33 & 8 & 26,62 \\
\hline $\begin{array}{l}\text { Glicose de Jejum } \\
(\geq 110 \mathrm{mg} / \mathrm{dl})\end{array}$ & - & - & 3 & 25,00 & 3 & 11,11 \\
\hline
\end{tabular}

\section{DISCUSSÃO}

As circunferências de cintura obtidas entre os indivíduos JBC, estatisticamente inferiores aos NJBC, demonstram um menor nível de deposição de gordura central entre os primeiros, sendo esta uma medida sugerida na literatura para determinação do depósito de gordura corporal ${ }^{(2)}$. Chan et al. ${ }^{(26)}$ correlacionaram circunferência de cintura com a predição de tecido adiposo em compartimentos, obtendo valores entre 0,66 e 0,85 dependendo do local de deposição da gordura.

Entretanto, a localização da deposição de gordura subcutânea não pode ser determinada por medidas circunferenciais, sugerindo uma metodologia adequada para o controle da obesidade subcutânea.

Os valores obtidos para PAS e PAD corroboram os achados de Haddad et al.(27), que verificaram respostas adaptativas da pressão arterial sistólica entre deficientes sob aumento no nível do condicionamento cardiovascular, sugerindo um modelo terapêutico importante para a resposta hipertensiva em deficientes físicos. Midha et al. ${ }^{(28)}$ em estudo sobre os efeitos do exercício no condicionamento físico e na função metabólica, não observaram diferença significativa da pressão arterial diastólica entre indivíduos portadores de lesão física, evidência esta também obtida neste estudo.

Ainda que os achados de pesquisa possam apresentar evidências discordantes sobre a resposta pressórica ao modelo de exercício físico, autores como Dela et al. ${ }^{(29)}$ e Steinberg et al. ${ }^{(30)}$, sugerem que o papel fundamental para o controle cardiovascular é o nível da lesão e as respostas humorais estabelecida por feedback, considerando os menores níveis de epinefrina e norepinefrina em indivíduos paraplégicos.

As evidências obtidas da glicemia sangüínea significativamente menor entre os JBC comparada aos NJBC, reforçam os achados de Phillips et al. ${ }^{(31)}$ sobre o modelo de exercício físico para o controle da glicemia sangüínea. Complementarmente, Vidal et al. ${ }^{(32)}$ incorpora a relação entre o grau de comprometimento da lesão, condição física e desajustes glicêmicos.

Alterações indesejáveis nos valores de glicemia sangüínea entre indivíduos com lesão física foram descritas por Jones, Legge, Goulding ${ }^{(33)}$, e outros estudos de revisão e intervenção têm demonstrado o efeito positivo do exercício físico na ação da insulina e conseqüente controle da glicemia, na adaptação aguda e crônica ${ }^{(31,34,35)}$.

Além das alterações da glicemia entre os NJBC, estes apresentaram níveis séricos desfavoráveis e significativamente superiores de CT, TG e LDL-C, comparados ao JBC. Presente em ambos os grupos, foi a dislipidemia da fração de colesterol HDL-C, discordando dos achados em estudos sobre atividade física e aumento desta fração de colesterol $^{(36,37)}$.

O perfil bioquímico sangüíneo dos indivíduos lesionados fisicamente sofre alterações pronunciadas do componente lipidêmico, tendo como uma das causas, o menor nível de mobilidade ou mesmo a ausência total de movimentos, postulando um papel adjuvante do exercício físico neste controle, muito embora outras variáveis devam ser consideradas como a etnia, dieta e o tabagismo ${ }^{(7,32,36,38,39)}$.

O controle do aporte diário de gorduras na ingesta caloria diária, parece uma medida apropriada para melhor identificar o papel do exercício físico sobre o perfil lipidêmico, excluindo a interferência nutricional, especialmente sobre TG e CT.

Os achados de Silva et al. ${ }^{(40)}$ corroboram os dados obtidos no presente estudo referentes ao HDL-C, embora uma mensuração acurada da intensidade, duração e freqüência do exercício físico tem sido reportada como mediador das adaptações favoráveis do HDL-C em grupos de indivíduos ativos ${ }^{(38)}$.

Uma mensuração acurada e amostra mais homogênea quanto ao tipo e nível de lesão, podem fornecer evidências sobre as relações 
entre exercício físico e fração lipoprotéica HDL-C na população de deficientes físicos.

A prevalência de fatores de risco para síndrome metabólica neste estudo foi significativamente elevada entre os NJBC, corroborando as evidências que apontam o exercício físico como medida adequada para a prevenção desta síndrome ${ }^{(41,42)}$.

Os modelos de exercício físico incluem atividades de caráter cardiovascular e musculoesquelético, com resultados reportados para uma ampla gama de intensidades e freqüências ${ }^{(43,44)}$. Ainda na prevenção e controle da síndrome metabólica a dieta parece desempenhar papel adjuvante, especialmente sob os quadros dislipidêmicos ${ }^{(45)}$.

Recentes estudos apontam para uma complexa interação de fatores de risco que determinam a síndrome metabólica, caracterizada por depósito de gordura central, dislipidemia, pressão arterial alta e níveis glicêmicos inadequados $(15,45,46)$.

Na amostra estudada, o fator de risco de maior prevalência foi a hipertensão arterial e adiposidade central no grupo de NJBC, e associadamente nos dois grupos, a dislipidemia com redução do HDL-C.

\section{CONCLUSÃO}

Considerando os menores níveis de deposição de gordura central, o aprimorado controle glicêmico e as alterações menos pronunciadas do perfil lipidêmico apresentados pelo grupo de jogadores comparado aos não jogadores, ambos portadores de deficiência física, sugerem um papel importante do exercício físico regular para o decréscimo da prevalência dos fatores de risco para a síndrome metabólica nesta população.

Estudos com uma amostra superior à estudada podem permitir inferências estatísticas e correlações entre tipos de lesão física e modelos diferenciados de exercício físico.

\section{AGRADECIMENTOS}

A indústria LabTest pelo apoio no fornecimentos dos kits de reagentes para realização das análises laboratoriais e ao Laboratório de Análises Clínicas do Centro Universitário de Patos de Minas - UNIPAM.

Todos os autores declararam não haver qualquer potencial conflito de interesses referente a este artigo.

\section{REFERÊNCIAS BIBLIOGRÁFICAS}

1. Spungen AM, Adkins RH, Stewart CA, Wang J, Pierson RN, Waters RL, et al. Factors influencing body composition in persons with spinal cord injury: a cross-sectional study. J Appl Physiol 2003; 95: 2398-407.

2. Janssen I, Katzmarzyk PT, Ross R. Body mass index, waist circunference, and health risk: evidence in support of current national institutes of health guidelines. Arc Intern Med 2002; 162: 2074-9.

3. Vidal J, Javierre C, Segura R, Lizarraga A, Barbany JR, Pérez A. Physiological adaptations to exercise in people with spinal cord injury. J Physiol Biochem 2003; 59: 11-8.

4. Ghorayeb N, Carvalho T, Lazzoli JK. Atividade física não competitiva para a população. In: Ghorayeb $\mathrm{N}$, editores. O exercício: preparação fisiológica, avaliação médica e aspectos especiais e preventivos. São Paulo: Editora Atheneu, 2004; 249-59.

5. DeVivo MJ, Black KJ, Stover SL. Causes of death during the first 12 years after spinal cord injury. Arch Phys Med Rehabil 1993; 74: 248-54

6. Buchholz AC, Bugarestti JM. A review of body mass index and waist circumference as markers of obesity and coronary heart disease risk in persons with chronic spinal cord injury. Spinal Cord 2005; 43: 513-8.

7. Moussavi RM, Cardus FR, Rintala DH, Rodriguez GP. Dietary and serum lipids in individuals with spinal cord injury living in community. Journal Rehab Res Dev 2001; 38: 225-33.

8. Sekhon LHS, Fehlings MG. Epidemiology, Demographics, and Pathophysiology of Acute Spinal Cord Injury. Spine 2001; 26: S2-12.

9. Garnshick E, Kelley A, Cohen SA, Garrison A, Tun CG, Gagnon D, et al. A prospective assessment of mortality in chronic spinal cord injury. Spinal Cord 2005; 43: 408-16.

10. Paffenbarger RS, hyde RT, Wing AL, Hsieh CC. Physical Activity, all-cause mortality, and longevity of college alumini. The New England Journal of Medicine 1986; 314: 605-13.

11. American College of Sports Medicine - ACSM. Position stand the recommended quantity and quality of exercise for developing and maintaining cardiorespiratory and muscular fitness, and flexibility in healthy adults. Med Sci Sports Exerc 1998; 30: 975-91.

12. Winett RA, Carpinelli RN. Potential health-related benefits of resistance training. Preventive Medicine 2001; 33: 503-13.

13. Lakka TA, Laaksonen DE, Lakka HM, Männikko N, Niskanen LK, Rauramaa R, et al. Sedentary Lifestyle, Poor Cardiorespiratory Fitness, and the Metabolic Syndrome. Med Sci Sports Exerc 2003; 35: 1279-86.

14. Barlow CE, Fitzgerald SJ, Jurca R, Church TS, Blair ST. Cardiorespiratory Fitness as a Predictor of Incident Metabolic Syndrome: Aerobics Center Longitudinal Study. Med Sci Sports Exerc 2004; 36: S7.

15. Grundy SM, Brewer HB, Cleeman JI, Smith Jr SC, Lenfant C. Definition of metabolic syndrome. Report of the American Heart Association/National Heart, Lung and Blood Institute/American Diabetes Association Conference on Scientific Issues Related to Management. Circulation 2004; 109: 433-8.

16. Wild S, roglic G, Green A, Sicree R, King H. Global Prevalence of Diabetes: Estimates for the year 2000 and projections for 2030. Diabetes Care 2004; 27: 1047-53.

17. Heyward VH, Stolarczyk LM. Avaliação da composição corporal aplicada. 1ed. Barueri: Manole, 2000. p. 2-98. Título do original: Applied body composition assessment.

18. Sociedade Brasileira de Cardiologia - SBC. IV Diretrizes Brasileiras de Hipertensão Arterial. Arq Bras Cardiol 2004: 82: S1-14.

19. Schmitz TJ. Sinais vitais. In: Sullivan SB, Schmitz TJ. (Ed.). Fisioterapia: avaliação e tratamento. 2ed. Barueri: Manole, 2004: 71-100

20. Motta VT. Bioquímica clínica para o laboratório: princípios e interpretações. 4ed. São Paulo: Robe Editorial, 2003: 43-158

21. Friedwald WT, Levy RI, Fredrickson DS. Estimation of the Concentration of Low-Density Lipoprotein Cholesterol in Plasma, Without Use of the Preparative Ultracentrifuge. Clin. Chem 1972; 18: 499-502.

22. Jekel JF, Katz DL, Elmore JG. Epidemiologia, bioestatística e medicina preventiva. 2ed. Porto Alegre: Artmed; 2005. p. 145-204. Título do original: Epidemiology, bioestatistics and preventive medicine.

23. Sociedade Brasileira de Cardiologia - SBC. I Diretriz Brasileira de Diagnóstico e tratamento da síndrome metabólica. Arq Bras Cardiol 2005; 84 Suppl 1: 1-28.
24. American Diabetes Association - ADA. Position statement: screening for type 2 diabetes. Diabetes Care 2003; 26: S21-4

25. Sociedade Brasileira de Cardiologia - SBC. III Diretrizes de Dislipidemias e Prevenção da Aterosclerose. Arq Bras Cardiol 2001; 77: 51-48

26. Chan DC. Waist circumference, waist-to-hip ratio and body mass index as predictors of adipose tissue compartments in men. Q J Med 2003; 96: 441-7.

27. Haddad S, Silva PRS, Barretto ACP, Ferraretto I. Efeito do treinamento físico de membros superiores aeróbio de curta duração no deficiente físico com hipertensão leve. Arq Bras Cardiol 1997; 69: 169-73.

28. Midha M, Schmitt JK, Sclater M. Exercise effect with the wheelchair aerobic fitness trainer on conditioning and metabolic function in disabled persons: a pilot study. Arch Phys Med Rehabil 1999; 80: 258-61.

29. Dela F, Mohr T, Jensen CMR, Haahr HL, Secher NH, Biering-Sorensen F, et al. Cardiovascular control during exercise: insights from spinal cord-injured humans. Circulation 2003; 107: 2127-33.

30. Steinberg LL, Lauro FAA, Sposito MMM, Tuffik S, Mello MT, Naffah-Mazzacorati MG, et al. Catecholamine response to exercise in individuals with different levels of paraplegia. Brazilian Journal of Medical and Biological Research 2000; 33: 913-8.

31. Phillips SM, Stewart BG, Mahoney DJ, Hicks AL, McCartney N, Tang JE, et al. Body-weight-support treadmill training improves blood glucose regulation in persons with incomplete spinal cord injury. J Appl Physiol 2004; 97: 716-24.

32. Vidal J, Javierre C, Curià Fj, Garrido E, Lizarraga MA, Sugura R. Long-term evolution of blood lipid profiles and glycemic levels in patients after spinal spinal cord injury. Spinal Cord 2003; 41: 178-81.

33. Jones LM, Legge M, Goulding A. Factor analysis of the metabolic syndrome in spinal cord-injured men. Metabolism 2004; 53: 1372-7.

34. Henriksen EJ. Exercise effects of muscle insulin signaling and action. Invited review: effects of acute exercise and exercise training on insulin resistance. J Appl Physiol 2002; 93: 788-96.

35. Houmard JA, Tanner CJ, Slentz CA, Duscha BR, McCartney JS, Kraus WE. The effect of the volume and intensity of exercise training on insulin sensitivity. J Appl Physiol 2004; 96: 101-6.

36. Dallmeijer AJ, Hopman MTE, Woude LHV. Lipid, lipoprotein, and apolipoprotein profiles in active and sedentary men with tetraplegia. Arch Phys Med Rehabil 1997; 78: 1173-6.

37. Washburn RA, Figoni SF. High density lipoprotein cholesterol in individuals with spinal cord injury: the potential role of physical activity. Spinal Cord 1999; 37: 685-95.

38. Bauman WA, Adkins RH, Spungen AM, Herbert R, Schechter C, Smith D, et al. Is imobilization associated with an anbnormal lipoprotein profile? Observations from a diverse cohort. Spinal Cord 1999; 37: 485-93.

39. Storch MJ, König D, Bültermann D, Blum A, Vogt S, Baumstark M, et al. Lipid profile in spinal cordinjured women with different injury levels. Preventive Medicine 2005; 40: 321-5.

40. Silva RC, Tirapegui J, Ribeiro SML, Pires ISO. Estudo controlado da influência da atividade física em fatores de risco para doenças crônicas em indivíduos lesados medulares paraplégicos do sexo masculino. Rev Bras Educ Fís Esp., 2004: 18: 169-77.

41. Laaksonen DE, Lakka HM, Salonen JT, Niskanen LK, Rauramaa R, Lakka TA. Low levels of leisure-time physical activity and cardiorespiratory fitness predict development of the metabolic syndrome. Diabetes Care 2002; 25: 1612-8.

42. Rennie KL, McCarthy N, Yazdgerdi S, Marmot M, Brunner E. Association of the metabolic syndrome with both vigorous and moderate physical activity. International Journal of Epidemiology 2003: 32: 600-6.

43. Durán FS, Lugo L, Ramírez L, Eusse E. Effects of an exercise program on the rehabilitation of patients with spinal cord injury. Arch Phys Med Rehabil 2001; 82: 1349-54

44. Bizzarini E, Saccavini M, Lipanje F, Magrin P, Malisan C, Zampa A. Exerise prescription in subjects with spinal cord injuries. Arch Phys Med Rehabili 2005; 86: 1170-5.

45. Manns PJ, McCubbin JA, Williams DP. Fitness, inflammation, and metabolic syndrome in men with paraplegia. Arch Phys Med Rehabil 2005; 86: 1176-81.

46. Carr DB, Utzschneider KM, Hull RL, Kodama K, Retzlaff BM, Brunzell JD, et al. Intra-abdominal fat is a major determinant of he National Cholesterol Education Program Adult treatment panel III criteria for the metabolic syndrome. Diabetes 2004; 53: 2087-94. 